\title{
Downregulation of hepatic lipase is associated with decreased CD133 expression and clone formation in HepG2 cells
}

\author{
XUEHUA LIU ${ }^{1 *}$, JUNHUA ZUO $^{2 *}$, YUAN FANG $^{1 *}$, JING WEN $^{1}$, FEIHONG DENG ${ }^{1}$, \\ HUI ZHONG ${ }^{1}$, BO JIANG ${ }^{3}$, JIDE WANG ${ }^{1}$ and BIAO NIE ${ }^{4}$ \\ ${ }^{1}$ Guangdong Provincial Key Laboratory of Gastroenterology, Department of Gastroenterology, Nanfang Hospital, \\ Southern Medical University, Guangzhou, Guangdong 510515; ${ }^{2}$ Department of Gastroenterology, \\ The Fifth Affiliated Hospital of Southern Medical University, Guangzhou, Guangdong 510900; \\ ${ }^{3}$ Department of Gastroenterology, Beijing Tsinghua Changgung Hospital, Tsinghua University, Beijing 102218; \\ ${ }^{4}$ The First Affiliated Hospital of Jinan University, Guangzhou, Guangdong 510630, P.R. China
}

Received February 23, 2016; Accepted May 7, 2018

DOI: $10.3892 / \mathrm{ijmm} .2018 .3756$

\begin{abstract}
The drug resistance of cancer remains a major obstacle to successful chemotherapy. New strategies for improving chemotherapeutic efficacy are urgently required. Recent studies have indicated that LIPC plays a role in promoting the liver metastasis of colorectal cancer. In the present study, we aimed to investigate the effects of LIPC on theproliferation and clone formation of colorectal cancer-derived cells, and chemoresistance in hepatoblastoma-derived HepG2 cells. The activity and expression of LIPC were determined in the cell lines by RT-qPCR and western blot analysis. HepG2 cells in which LIPC was knocked down by LIPC short hairpin RNA (shRNA) and control cells [shRNA control (shCON)] were established and analyzed for cell proliferation and colony formation rates. FACS analysis was used to explore the association between LIPC and the tumor-derived cell biomarker, CD133, and the percentages of CD133-positive cells were assessed by FACS. Additionally, shLIPC- and shCON-transfected cells were treated with various concentrations of doxorubicin and 5-floxuridine (5-FU), and cell viability was determined by MTT assay. mRNA levels in the shLIPC- and shCON-transfected cells were compared by cDNA microarray and Kyoto Encyclopedia of Genes and Genomes (KEGG) pathway analysis. The results revealed that the HepG2 cells exhibited a relatively higher LIPC activity
\end{abstract}

Correspondence to: Dr Biao Nie, The First Affiliated Hospital of Jinan University, Guangzhou, Guangdong 510630, P.R. China

E-mail: niebiao2@163.com

Professor Jide Wang, Guangdong Provincial Key Laboratory of Gastroenterology, Department of Gastroenterology, Nanfang Hospital, Southern Medical University, Guangzhou, Guangdong 510515, P.R. China

E-mail: jidewang55@163.com

${ }^{*}$ Contributed equally

Key words: hepatic lipase, hepatocellular carcinoma, CD133, clone formation, cell adhesion molecules and expression levels compared to the other colon cancer cell lines. The downregulation of LIPC in the HepG2 cells was associated with the decreased expression of CD133, decreased cell proliferation and colony formation, as well as increased resistance to chemotherapy. KEGG analysis of the cDNA microarray data revealed increased levels in the cell adhesion molecule (CAM) pathway, including CLDN10 and CLDN1, indicating that CAMs may play a role in LIPC-mediated tumor progression. The present findings indicate a potential role of LIPC as a promising therapeutic target in cancer.

\section{Introduction}

Liver cancer is one of the most prevalent types of cancer. Hepatoblastoma is a malignant liver cancer with a poor prognosis and is most commonly diagnosed in the first 3 years of life of children. Moreover, hepatocellular carcinoma (HCC) is the third leading cause of cancer-related mortality worldwide (1). This statistic is attributed to the fact that a large number of patients are diagnosed with HCC at an advance stage, which leads to a poor prognosis. For these patients, systemic chemotherapy is considered an alternative option. However, HCC is relatively chemotherapy-resistant and patients receiving chemotherapy still have an unsatisfactory prognosis and a high rate of recurrence (2). Some studies have demonstrated that HCC resistance to chemotherapy may be due to the presence of hepatic tumor-derived cells, which are commonly considered to be responsible for tumor initiation, self-renewal, metastasis and chemoresistance (3). Therefore, a greater understanding of the mechanisms of tumor chemoresistance and potential molecular targets is urgently required.

Hepatic lipase (LIPC), a member of the lipase gene family, is a hydrolytic enzyme with lipolytic and ligand function and plays an important role in lipoprotein metabolism and cytokine homeostasis (4). LIPC has been reported to be closely associated with coronary artery disease and diabetes (5). Recent studies have demonstrated the effects of LIPC in cancer progression and metastasis. A previous study suggested that monoacylglycerol lipase (MAGL) promotes tumor cell proliferation, invasion and migration by regulating a fatty acid network with carcino- 
genesis signaling molecules (6). Another study by Ding et al reported that apolipoprotein $B$ mRNA editing enzyme catalytic polypeptide-like 3G (APOBEC3G), S100P, LIPC and CD133 played a critical role in promoting liver metastasis of colorectal cancer, and could be potential markers for predicting the likelihood of hepatic metastasis (7). More recently, another study indicated that the intratumoral level of LIPC positively correlated with the prognosis of non-small cell lung carcinoma (NSCLC), and patients with relatively low expression of LIPC may benefit from chemotherapy (8). However, the precise role of LIPC in tumor development and chemotherapy remains unknown. CD133 has been commonly considered as a marker of tumor-derived cells in various tumors including liver cancer and colon cancer. It has been also found to be involved in chemoresistance $(9,10)$. Ding et al demonstrated that both LIPC and CD133 promote the liver metastasis of colorectal cancer (7). However, no evidence has been provided as to whether they function interactively or independently in tumors.

In this study, we aimed to investigate the role of LIPC in cancer cells and to elucidate the potential underlying mechanisms. We demonstrate that the downregulation of LIPC expression significantly decreases cell proliferation and the colony formation rate of HepG2 cells. The expression of CD133 significantly decreased in short hairpin RNA (shLIPC)-transfected HepG2 cells compared with control short hairpin RNA (shCON)-transfected HepG2 cells. The knockdown of LIPC increased resistance to doxorubicin and 5-floxuridine (5-FU). Microarray analysis of gene expression profiles in the shCON/shLIPC-transfected HepG2 cells identified differentially expressed genes for further analysis.

\section{Materials and methods}

Cell lines, cell culture and reagents. All cells [human colorectal cancer cells SW620 (CCL-227), SW480 (CCL-228), LoVo (CCL-229), LS174T (CL-188), HCT 116 (CCL-247), HT29 (HTB-38), hepatoblastoma cells HepG2 (HB-8065), and mouse colorectal cancer cells CT26 (CRL-2638)] were purchased from the American Type Culture Collection (ATCC, Manassas, VA, USA). The cells were grown in Dulbecco's modified Eagle's medium (DMEM; Gibco, Grand Island, NY, USA), supplemented with $10 \%$ fetal bovine serum (FBS). The cells were maintained at $37^{\circ} \mathrm{C}$ under $5 \% \mathrm{CO}_{2}$. Doxorubicin and 5-FU were dissolved in dimethyl sulfoxide (DMSO) (all from Sigma (St. Louis, MO, USA) and stored as $10 \mathrm{mmol} / \mathrm{l}$ stock solutions in the dark at $-20^{\circ} \mathrm{C}$.

LIPC activity assay. The cell culture supernatants were collected and the activity of LIPC was measured using a human hepatic triglyceride lipase (HTGL) enzyme linked immunosorbent assay (ELISA) kit (Cusabio, Wuhan, China) according to the manufacturer' $\mathrm{s}$ instructions.

$R N A$ extraction and $R T-q P C R$. Total RNA was extracted from the cells using TRIzol reagent (Invitrogen, Carlsbad, CA, USA) and First Strand cDNA synthesis was performed with a PrimeScript ${ }^{\mathrm{TM}}$ RT reagent kit (DRR037A; Takara, Shiga, Japan). qPCR was performed using SYBR-Green Premix Ex Taq (RR091A; Takara) and analyzed with the LC480 system (Roche, Mannheim, Germany). Specific primer sequences were designed using NCBI primerblast for: LIPC, 5'-TCC CAA AGT ACC CAA AGG C-3' (sense) and 5'-ACT CCA GCC TTG ACC CAC TC-3' (antisense); glyceraldehyde phosphate dehydrogenase (GAPDH), 5'-TGG AAG GAC TCA TGA CCA CA-3' (sense) and 5'-TTC AGC TCA GGG ATG ACC TT-3' (antisense). The qPCR conditions were $95^{\circ} \mathrm{C}$ for $5 \mathrm{~min}$, followed by 45 cycles of $95^{\circ} \mathrm{C}$ for $10 \mathrm{sec}, 58^{\circ} \mathrm{C}$ for $30 \mathrm{sec}$, and $72^{\circ} \mathrm{C}$ for $20 \mathrm{sec}$. A final extension at $72^{\circ} \mathrm{C}$ for $5 \mathrm{~min}$ was included before a temperature ramp from 72 to $95^{\circ} \mathrm{C}$ at $0.1^{\circ} \mathrm{C} / \mathrm{sec}$. GAPDH was used as an internal reference gene, and the $2^{-\Delta \Delta \mathrm{Cq}}$ method (11) was used to calculate cycle threshold values. All experiments were repeated at least 3 times and the data are presented as mean \pm standard deviation $(\mathrm{SD})$.

Western blot analysis. Total cellular protein was extracted from the cells using RIPA buffer containing $1 \mathrm{X}$ protease inhibitor cocktail (Thermo Fisher Scientific, Waltham, MA, USA) and 1X PMSF. The protein concentration was determined using a BCA protein assay kit (Beyotime Institute of Biotechnology, Shanghai, China). Forty micrograms of protein lysate were separated by $10 \%$ sodium dodecyl sulfate-polyacrylamide gel electrophoresis (SDS-PAGE) electrophoresis and transferred onto PVDF membranes (Millipore, Billerica, MA, USA). Mouse anti-human LIPC (1:500, sc-21740) and mouse antihuman $\beta$-actin $(1: 5,000$, sc-47778) (both from Santa Cruz Biotechnology, Inc., Santa Cruz, CA, USA) were used as primary antibodies, followed by incubation with a secondary antibody (goat anti-mouse IgG-HRP; 1:5,000, sc-2005; Santa Cruz Biotechnology, Inc.). After washing, the immunoreactive bands were detected by enhanced chemiluminescence (ECL) reagents (Millipore).

Lentiviral transduction and construction of stable cell lines. We selected the HepG2 cell line for shRNA interference assays as the HepG2 cells were found to have a high expression of LIPC. For the knockdown experiments, a LIPC siRNA lentiviral vector and a control vector were constructed (GenePharma Co., Ltd., Shanghai, China). The mouse LIPC gene sequence (NM-008280) was amplified by PCR and cloned into the pCMV6-Kan/Neo plasmid (OriGene, Rockvile, MD, USA). A specific LIPC siRNA sequence was designed as: 5'-GGA GAA ACC CAG CAA AGA AdTdT-3' (10). Both constructs were confirmed by DNA sequencing before use. To make the lentiviral particle, LIPC/control shRNA plasmid constructs were co-transfected with the helper plasmids pGag/ Pol, pRev and pVSV-G into 293T cells (purchased from ATCC) using RNAi-Mate transfection reagent (GenePharma Co., Ltd.) according to the manufacturer's instructions. The viral particles were collected and the lentiviral titer was determined by quantifying the EGFP-positive cells by flow cytometry analysis following infection of the $293 \mathrm{~T}$ cells. To prepare stably transfected cell lines, HepG2 cells at 40-60\% confluence (in 24-well plates) were transfected with moieties of infection (MOI) of 10, in the presence of $5 \mu \mathrm{g} / \mathrm{ml}$ polybrene (Sigma). G418 was used to select cells at a concentration of $400 \mathrm{~g} / \mathrm{ml}$. The efficiency of transfection was determined by measuring the LIPC levels by western blot analysis after 48-72 h.

Flow cytometric analysis of CD133 expression. To determine the expression of the tumor-derived cell biomarker, CD133, 
single cell suspensions were collected and re-suspended in $200 \mu \mathrm{l}$ at a density of $1 \times 10^{6}$ cells $/ \mathrm{ml}$ in phosphate-buffered saline (PBS) containing $0.1-0.5 \%$ bovine serum albumin (BSA). The cells were incubated with antigen presenting cell $x(\mathrm{APC})-$ conjugated anti-human CD133 (130-090-826; Miltenyi Biotec, Bergisch Gladbach, Germany) for $30 \mathrm{~min}$ at $4^{\circ} \mathrm{C}$ in the dark. Cells incubated with anti-mouse-IgG (sc-2005; Santa Cruz Biotechnology, Inc.) were used as the isotype control. The cells were washed with pre-chilled PBS twice and re-suspended for flow cytometric analysis. For detecting CD133 expression in the cytoplasm, the cells were first fixed with $100 \mu \mathrm{l}$ $1 \%$ permeabilization buffer in PBS before staining with CD133 antibodies. The assay was repeated 3 times.

Cell proliferation and colony formation assay. The HepG2 cells transfected with shLIPC or shCON vectors were seeded into a 96-well plate at a density of 5,000 cells/well and incubated for $72 \mathrm{~h}$. Subsequently, $20 \mu \mathrm{l}$ of 3-(4,5-dimethylthiazol-2-yl)2,5-diphenyltetrazolium bromide (MTT) $(0.5 \mathrm{mg} / \mathrm{ml})$ was added and incubated for another $4 \mathrm{~h}$ at $37^{\circ} \mathrm{C}$. The medium was aspirated and $150 \mathrm{ml}$ DMSO was added to dissolve the formazan crystals. After the crystals were completely dissolved, the absorbance was measured using a microplate reader (ELX800; Bio-Tek Instruments, Winooski, VT, USA). The number of viable cells was expressed as a percentage of absorbance. For colony formation assays, transfected cells were plated in triplicate into 6-well plates at a density of 200-800 cells/well and incubated for 14 days (12). After being washed and fixed with $70 \%$ methanol for $15 \mathrm{~min}$, the cells colonies were stained with $0.1-0.5 \%$ crystal violet for $10-15 \mathrm{~min}$. The plates were washed with water and left to dry. The number of colonies containing $>50$ cells was counted. The experiment was repeated 3 times.

Drug resistance. The cells were treated with or without doxorubicin $(0.03-0.3 \mu \mathrm{M})$ and 5-FU $(0.2-2.0 \mu \mathrm{M})$ and further incubated for $48 \mathrm{~h}$. The proliferation of both the shLIPC- and shCON-transfected HepG2 cells was determined by MTT assay as described above.

cDNA microarray analysis. Microarray experiments were performed by RiboBio Co., Ltd. (Guangzhou, China). Initially, total RNA of the shLIPC/shCON-transfected HepG2 cell lines was extracted and the quantity of RNA was determined using an Agilent 2200 Bioanalyzer (Agilent, Santa Clara, CA, USA). Three independent samples of each group were prepared and an equal amount of total RNA from each preparation was pooled, respectively. The CDNA was synthesized and labeled with Cy3/Cy5, and randomly hybridized to a RiboArray ${ }^{\mathrm{TM}}$ Custom array $1 \mathrm{x} 40 \mathrm{~K}$ (RiboBio, Guangzhou, China) according to the manufacturer's instructions. The hybridized microarray was scanned and the data were analyzed and normalized. The differentially expressed genes were identified at a fold change $\geq 2$.

GO analysis and pathway enrichment. The gene ontology (GO) and Kyoto Encyclopedia of Genes and Genomes (KEGG) pathway enrichment analysis of the differentially expressed genes were performed using DAVID tools.

Statistical analyses. Statistical analyses were performed using SPSS 20 software. All data were presented as the means \pm SD of three independent experiments. For cell functional assays, the 3 significance between the shLIPC and shCON groups were determined by a two-tailed unpaired Student's t-test. For chemoresistance experiments, data were analyzed with two-factor variance analysis and Student-Newman-Keuls (SNK) test. P-values $<0.05$ were considered statistically significant.

\section{Results}

Differential expression of LIPC in cancer cells. LIPC activity was examined in colorectal cancer cells (human SW620, SW480 and LoVo cells, and mouse CT26 cells) and hepatoblastoma HepG2 cells. The HepG2 cells exhibited a 1.92-fold higher LIPC activity compared to the other cells $(\mathrm{P}<0.001)$ (Fig. 1A). The LIPC mRNA levels in the HepG2 cells determined by RT-qPCR were the highest among the cell lines $(\mathrm{P}<0.001)$ (Fig. 1B). Similarly, the protein levels of LIPC in the HepG2 cells were significantly increased compared with the other cell lines $(\mathrm{P}<0.001)$ (Fig. 1C). The HepG2 cells exhibited relatively higher levels of LIPC compared to the other cell lines, and were thus selected as a suitable model for shRNA interference assays. The protein levels of LIPC were determined by western blot analysis. Compared with the shCON-transfected cells, the mRNA and protein levels of LIPC were significantly decreased in the shLIPC-transfected cells $(\mathrm{P}<0.001)$ (Fig. 1D).

Downregulation of LIPC decreases the expression of CD133. The association between LIPC and the tumor-derived cell marker, CD133, was explored. CD133 expression was decreased significantly in the HepG2 cells transfected with shLIPC $(4.62 \pm 0.36 \%)$ compared to the HepG2 shCONtransfected cells $(33.94 \pm 0.94 \%)(\mathrm{P}<0.001)$ (Fig. 2A, left panel). Similar results were observed for CD133 expression in the cytoplasm. The expression of CD133 in the cytoplasm of the HepG2 shLIPC-transfected cells $(14.11 \pm 0.57 \%)$ was significantly decreased compared to that of the HepG2 shCON-transfected cells $(50.35 \pm 0.97 \%)(\mathrm{P}<0.001)$ (Fig. $2 \mathrm{~A}$, right panel).

Downregulation of LIPC decreases cell proliferation and colony formation. MTT assays indicated that cell proliferation was slightly decreased after LIPC knockdown compared to the respective control groups $(\mathrm{P}<0.05)$ (Fig. 2B). We also performed a colony formation assay to analyze the proliferative potential of single cells after the silencing of LIPC in vitro. The size of single colony formation in shLIPC-transfected HepG2 cells was markedly smaller and the number of colonies containing $\geq 50$ cells was reduced by $64.4 \pm 2.7 \%$ compared to the shCON-transfected cells $(\mathrm{P}<0.001)$ (Fig. 2C). These data suggest that the knockdown of LIPC inhibits the proliferation and colony formation of HepG2 cells.

Downregulation of LIPC increases chemoresistance in HepG2 cells. Cells transfected with shLIPC exhibited an increased cell viability following treatment with $0.03,0.1$ and $0.3 \mu \mathrm{M}$ doxorubicin compared to the shCON-transfected cells $(\mathrm{P}<0.05)$ (Fig. 3A). Similar results were observed in cells treated with 0.2 and $2.0 \mu \mathrm{M} 5-\mathrm{FU}(\mathrm{P}<0.01)$ (Fig. 3B). The current data suggest that the downregulation of LIPC enhances resistance to doxorubicin and 5-FU in HepG2 cells. 

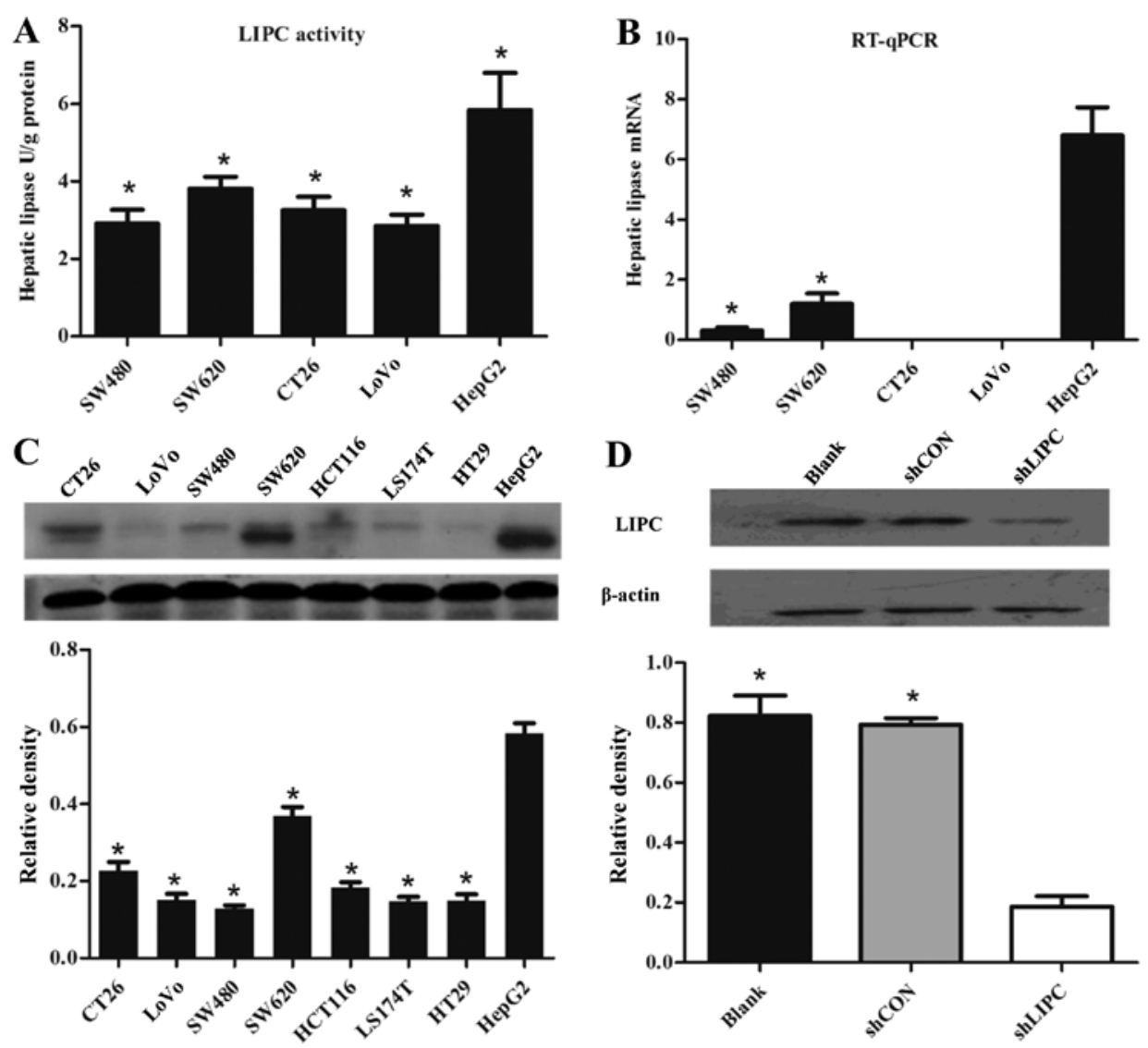

Figure 1. Determination of downregulated hepatic lipase (LIPC) expression by short hairpin RNA (shRNA) in HepG2 cells. (A) The levels of lipase activities in SW480, SW620, LoVo, CT26 and HepG2 cells were determined. (B) mRNA levels assayed by RT-qPCR. (C) Western blot analysis of the LIPC expression in various cancer cells. (D) Protein levels of LIPC determined by western blot analysis, respectively in blank control cells, shRNA control cells and LIPC shRNA cells. Data are presented as mean \pm SD from three independent experiments. ${ }^{*} \mathrm{P}<0.001$ compared to HepG2 cells.
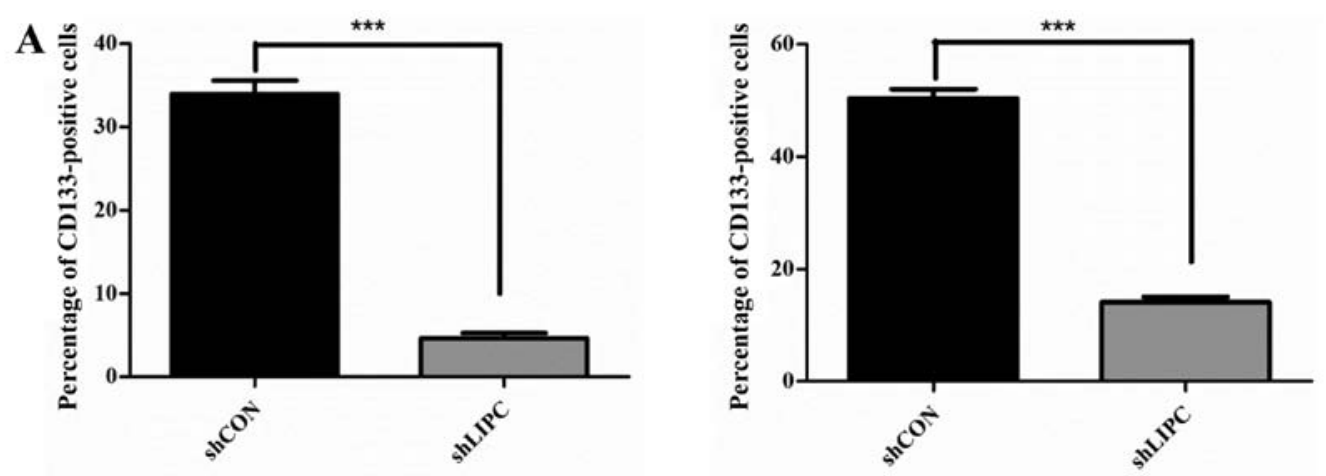

B
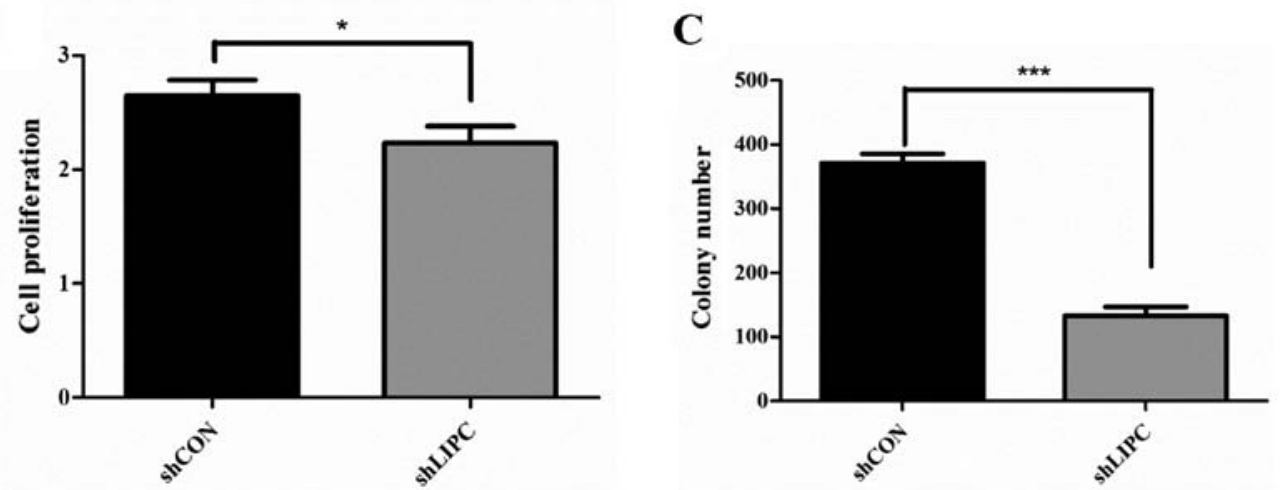

Figure 2. Effects of the knockdown of hepatic lipase (LIPC) on CD133 expression and colony formation. (A) The percentages of CD133-positive cells were determined by FACS with live cells (left panel) or perforated cells (right panel). (B) Cell proliferation was determined by MTT assay in short hairpin RNA control (shCON) and shLIPC HepG2 cells. (C) Colony formation assay was performed and the number of colonies containing $>50$ cells was counted after methyl violet staining. Data are presented as mean $\pm \mathrm{SD}$ from triplicate determinations. ${ }^{*} \mathrm{P}<0.05$ and ${ }^{* * * *} \mathrm{P}<0.001$. 

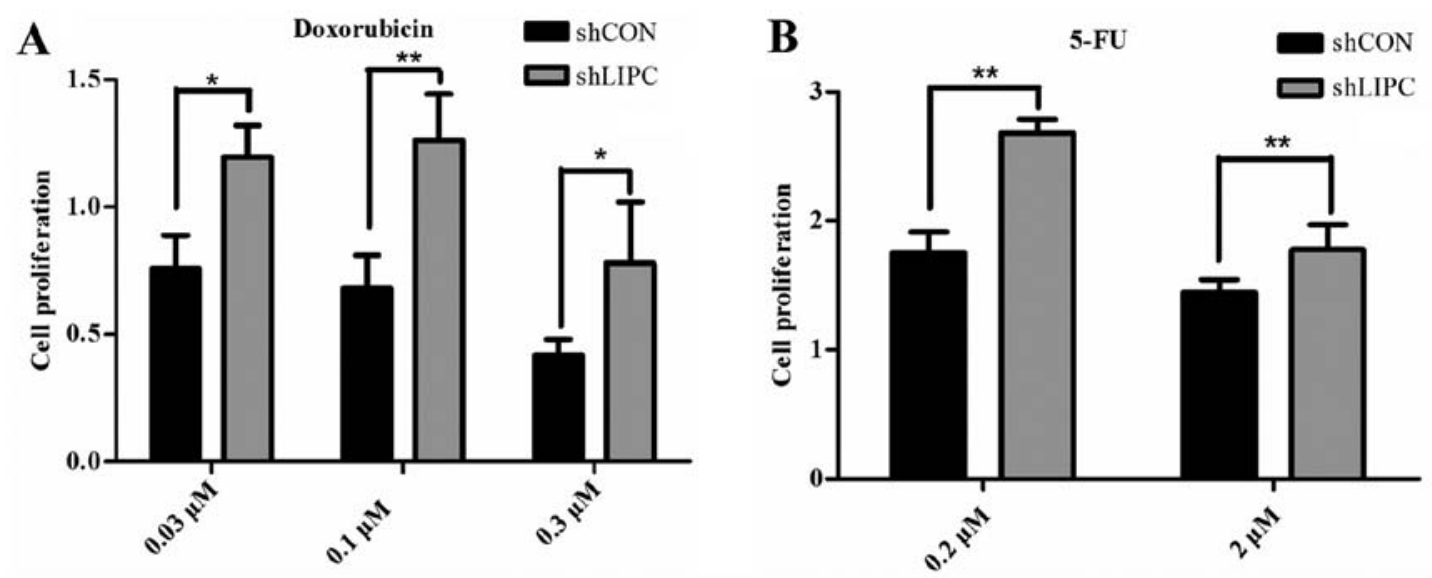

Figure 3. Effects of the knockdown of hepatic lipase (LIPC) on chemoresistance. The control short hairpin RNA (shRNA)-transfected cells and LIPC shRNAtransfected cells were treated with various concentrations of doxorubicin $(0.03,0.1$ and $0.3 \mu \mathrm{M})$ and 5-floxuridine $(5$-FU) $(0.2$ and $2.0 \mu \mathrm{M})$ and the cell proliferation was measured by MTT assay. Data are presented as mean \pm SD from triplicate determinations. ${ }^{*} \mathrm{P}<0.05$ and ${ }^{* *} \mathrm{P}<0.01$.

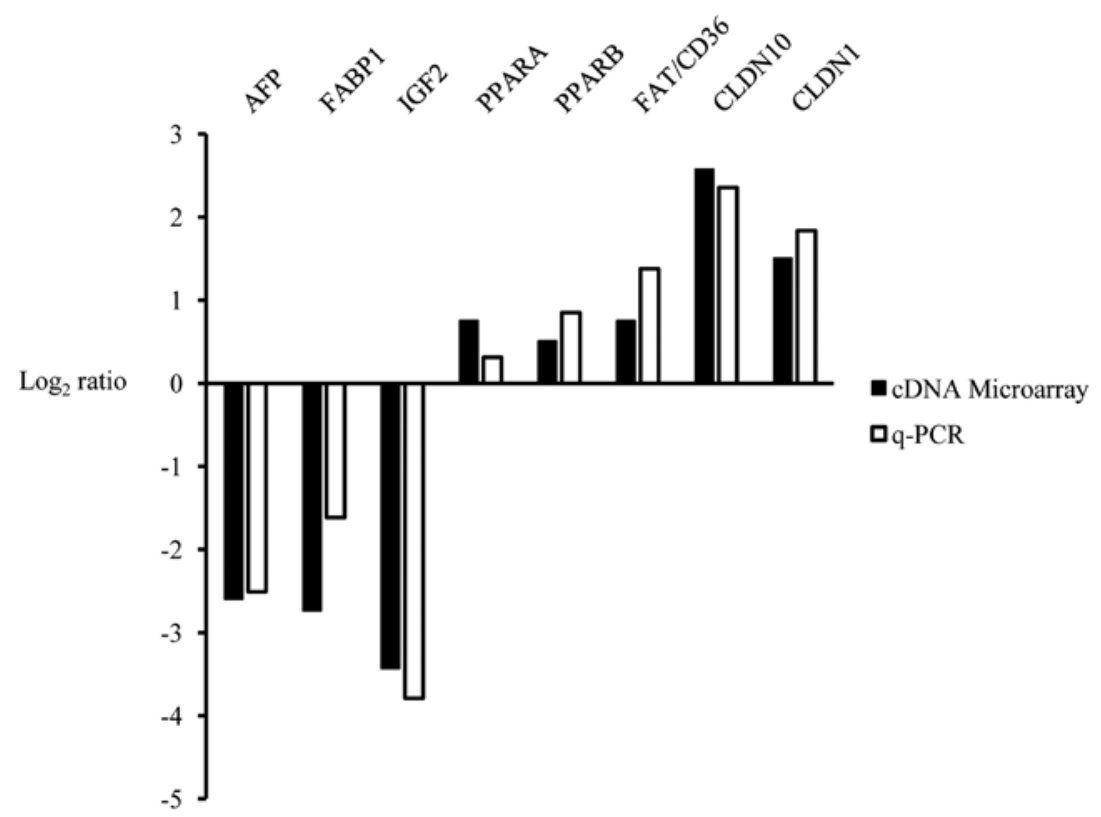

Figure 4. Gene analyses of HepG2-shLIPC from cDNA microarray. The mRNA levels of 8 genes containing 5 upregulated genes and 3 downregulated genes were validated by RT-qPCR.

We observed no significant differences in drug resistance assays following LIPC upregulation (data not shown).

Genes differentially expressed following the knockdown of LIPC. We found 1,602 genes that were upregulated and 716 genes that were downregulated in the shLIPC-transfected cells compared with the shCON-transfected cells using a $\log _{2}$ (fold change) $\geq 1$ as the standard selection criteria. Detailed gene lists of the top 25 of the most differentially expressed genes are summarized in Table I. Eight candidate genes from the microarray analysis were selected for validation by RT-qPCR (Fig. 4).

To examine the function of the differentially expressed mRNAs and identify the biological pathway involved, the data were further analyzed using Gene Ontology (GO) and the Kyoto Encyclopedia of Genes and Genomes (KEGG) database. GO analysis revealed that the differentially expressed genes fell into the following categories: Regulation of tran- scription, NA-dependent, signal transduction in biological process ontology; integral to membrane, nucleus and cytoplasm in cellular component ontology; and protein binding, zinc ion binding, and metal ion binding in molecular function ontology (Fig. 5). In KEGG analysis, a total of 124 pathways were regulated by the differentially expressed genes, which mainly involved cell adhesion molecules (CAMs), axon guidance, the vascular endothelia growth factor (VEGF) signaling pathway, and the Wnt signaling pathway. The top 12 regulated pathways are listed in Fig. 6.

\section{Discussion}

Little is known about the mechanisms of action of LIPC in cancer cells. Previous studies reported that serum lipase activities were elevated before detection of tumor relapse or progression (13-15). These findings suggest that lipase may play a tumor marker-like role and contributed to the early detection 
Table I. Top 25 upregulated and downregulated transcripts in HepG2 cells after the knockdown of hepatic lipase (LIPC).

\begin{tabular}{|c|c|c|c|c|c|c|c|}
\hline $\begin{array}{l}\text { Upregulated } \\
\text { accession }\end{array}$ & $\begin{array}{l}\text { Downregulated } \\
\text { gene ID }\end{array}$ & Gene symbol & Ratio & Accession & Gene ID & Gene symbol & Ratio \\
\hline NM_007342 & 11097 & NUPL2 & 22.08 & NM_001005482 & 79310 & OR5H2 & 12.12 \\
\hline NM_001024644 & 2829 & XCR1 & 17.16 & NR_038404 & 100506801 & LOC100506801 & 11.08 \\
\hline NR_037897 & 386627 & FLJ38109 & 14.97 & NM_001127598 & 3481 & IGF2 & 10.80 \\
\hline XR_132758 & 440157 & LOC440157 & 9.665 & NM_001135189 & 3267 & AGFG1 & 10.30 \\
\hline NM_181706 & 120526 & DNAJC24 & 9.400 & NM_182715 & 6856 & SYPL1 & 9.487 \\
\hline NR_029975 & 574413 & MIR409 & 8.755 & NM_021871 & 2243 & FGA & 8.808 \\
\hline NM_001214 & 750 & C16orf3 & 8.626 & NM_014628 & 9587 & MAD2L1BP & 7.579 \\
\hline NM_033306 & 837 & CASP4 & 8.566 & NM_004536 & 4671 & NAIP & 7.358 \\
\hline XR_159202 & 100506518 & LOC100506518 & 8.246 & NM_001443 & 2168 & FABP1 & 6.662 \\
\hline NM_001170796 & 79752 & ZFAND1 & 7.770 & NM_000509 & 2266 & FGG & 6.559 \\
\hline NM_007224 & 11247 & NXPH4 & 7.133 & NM_000612 & 3481 & IGF2 & 6.381 \\
\hline NM_003644 & 8522 & GAS7 & 6.800 & NM_001134 & 174 & AFP & 6.050 \\
\hline NM_001136508 & 284546 & C1orf185 & 6.701 & NM_001100912 & 222389 & BEND7 & 5.778 \\
\hline NM_022746 & 64757 & MARC1 & 6.611 & NR_003512 & 723961 & INS-IGF2 & 5.378 \\
\hline NR_037456 & 100500802 & MIR3685 & 6.551 & NM_019855 & 56344 & CABP5 & 5.132 \\
\hline XR_133419 & 100653086 & LOC100653086 & 6.527 & NM_000327 & 6094 & ROM1 & 5.128 \\
\hline NM_153448 & 80712 & ESX1 & 6.475 & NM_001007559 & 6760 & SS18 & 5.118 \\
\hline NM_206922 & 401262 & CRIP3 & 6.406 & NM_00101172 & 144983 & HNRNPA1L2 & 5.044 \\
\hline NM_014294 & 23471 & TRAM1 & 6.101 & NR_036141 & 100422870 & MIR3180-1 & 5.007 \\
\hline XM_003403794 & 100509542 & LOC100509542 & 6.034 & NR_033832 & 340113 & LOC340113 & 4.978 \\
\hline NM_015474 & 25939 & SAMHD1 & 6.015 & NM_000014 & 2 & $\mathrm{~A} 2 \mathrm{M}$ & 4.824 \\
\hline NM_006984 & 100500882 & CLDN10 & 5.983 & NM_001063 & 7018 & $\mathrm{TF}$ & 4.809 \\
\hline NR_037440 & 9071 & MIR3667 & 5.968 & NM_001025253 & 7163 & TPD52 & 4.751 \\
\hline NR_015364 & 441204 & LOC441204 & 5.911 & NM_001256654 & 7594 & ZNF43 & 4.493 \\
\hline NR_026562 & 55969 & C20orf24 & 5.853 & NR_001443 & 339240 & LOC339240 & 4.422 \\
\hline
\end{tabular}
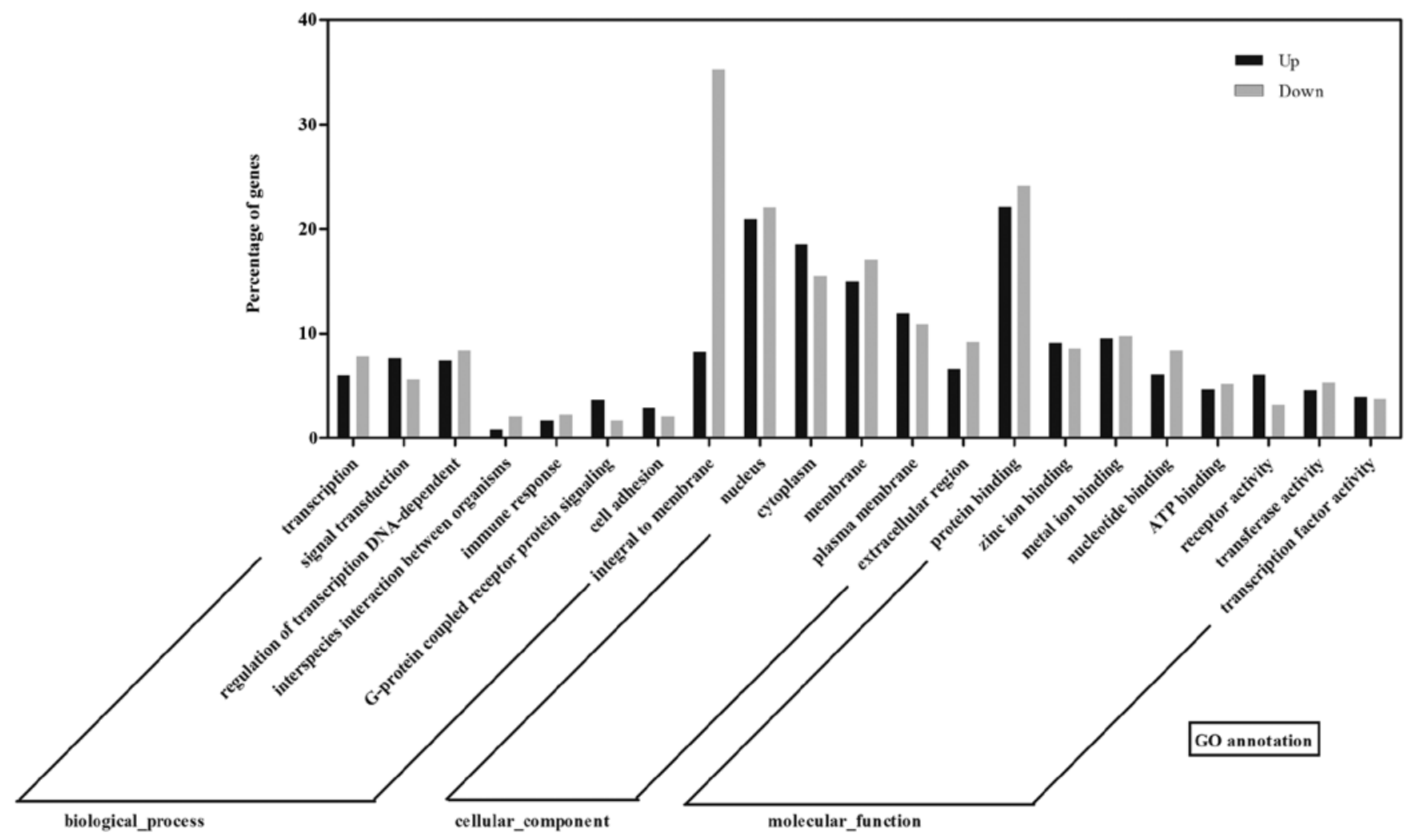

Figure 5. Gene Ontology (GO) analysis for the differentially expressed mRNA. Upregulated genes $(1,602)$ and downregulated genes $(716)$ were identified by cDNA microarray assay and further analyzed with a GO database. 


\section{KEGG pathway enrichment}

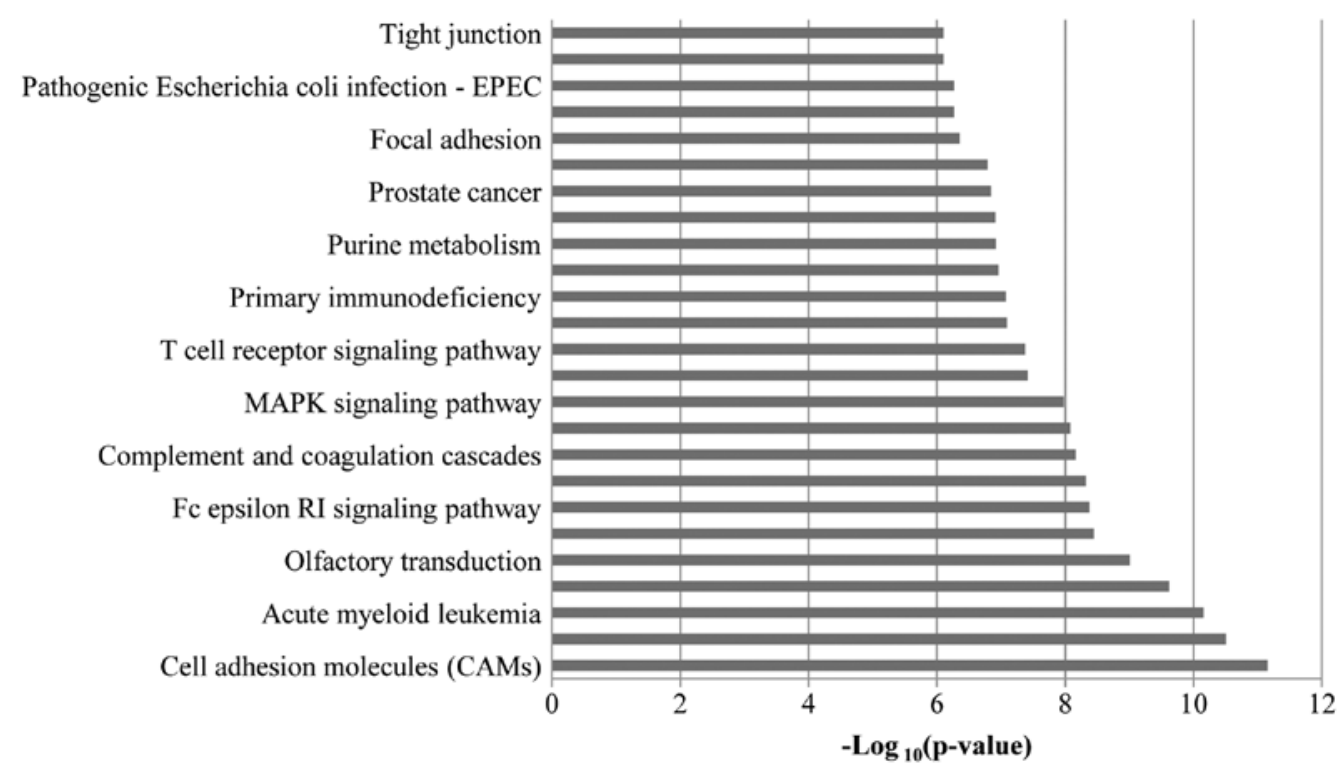

Figure 6. Kyoto Encyclopedia of Genes and Genomes (KEGG) pathway analysis for the differentially expressed mRNA. The top 12 regulated pathways were summarized.

of malignant neoplasm. In this study, we demonstrated that the downregulation of LIPC inhibited CD133 expression in and the colony formation of HepG2 cells.

CD133, also known as prominin-1, is considered a marker for a tumor-derived cell population of brain tumors (16), melanoma (17), prostate $(18,19)$, colon $(20,21)$ and liver cancers $(22,23)$. A number of studies have indicated that CD133(+) cells are capable of greater tumorigenicity and a higher incidence of metastasis compared to CD133(-) cells. O'Brien et al demonstrated that only CD133(+) colon cancer cells were able to initiate tumor growth in immunodeficient mice (20). Bao et al found that CD133(+) glioma cancer cells promoted tumor angiogenesis through VEGF (24). However, the role of CD133 as a tumorderived cell marker is controversial. In fact, a number of studies have indicated that the CD133(-) population is equally capable, if not more aggressive, of tumor initiation $(25,26)$. Jaksch et al argued that differential CD133 expression may be a marker of different cell cycle stages rather than a marker of stable tumorderived cells (27). Taken together, these studies suggest that too much attention has been placed on the role of CD133 for the identification of tumor-derived cells, while the actual biological functions of this molecule remain unknown. A previous study by Ding et al revealed that both LIPC and CD133 contributed to liver metastasis (7). The data from the present study revealed that a decreased CD133 expression was associated with LIPC deficiency in HepG2 cells, which was demonstrated to inhibit cell proliferation and decrease the colony formation rates.

However, in terms of chemoresistance, there was no result that was consistent with that of previous literature (8), since the reduction in CD133 expression did not decrease chemoresistance to doxorubicin and 5-FU. It might be that it is LIPC not CD133, which plays a role in regulating drug resistance. As regards the use of CD133 as a stable marker for tumorderived cells, some previous studies have indicated that the biological behavior of tumor-derived cells is influenced by the surrounding microenvironment, which is affected by tumor stages, histopathological types, nutritional conditions and therapies (28). We found that in LIPC-silenced cells, a higher percentage of 'membrane-integrated' genes were downregulated and more cell adhesion molecules were regulated. Under the conditions of cell culture, different surrounding microenvironments affecting cell adhesion in vitro might result in such drug resistance results. In the next step, the drug resistance in vivo is worth investigating.

One intriguing finding of the present study was that the CAM pathway was demonstrated to be the most significantly regulated pathway by the differentially expressed genes. Moreover, the mRNA levels of CLDN10 and CLDN1, which participate in the CAMs pathway, were found to be almost six times and three times higher in shLIPC cells compared to shCON cells, respectively. Some previous studies have indicated that a high expression of CLDN10 was associated with the recurrence of primary hepatic carcinoma, and CLDN10 enhanced cell invasiveness and motility $(29,30)$. Suh et al found that CLDN1 could promote metastasis of liver cancer by inducing epithelial-mesenchymal transition (EMT) (31). However, in the present study, we did not see obvious differences in cell migration after the knockdown of LIPC (data not shown). We speculate that the CAM pathway may have important implications for the mechanisms involved in the tumor colony formation induced by LIPC expression.

A limitation of our study was that we performed the experiments in a hepatoma cell in culture, which may present distinct biological features without the regulation of the surrounding microenvironment. Another limitation is that data on more cell lines were not provided in this study. Further studies using animal models and clinical tissue specimens are required in the future. The present findings reveal the potential role of LIPC as a therapeutic target in liver cancer. The association of LIPC and CD133 remains to be determined, and further studies are warranted to determine the specific mechanisms through which LIPC participates in tumor progression. 


\section{Acknowledgements}

The authors would like to acknowledge Medjaden Bioscience Limited for the English language editing service.

\section{Funding}

The study was sponsored by the Returned Scientific Research Foundation of the Ministry of Education and National Natural Science Foundation of China (grant no. 81471080) to B.N. This study was supported by the Guangzhou Pilot Project of Clinical and Translational Research Center (early gastrointestinal cancers, no. 7415696196402) and the Guangdong Provincial Bio-engineering Research Center for Gastroenterology Diseases.

\section{Availability of data and materials}

The datasets used and/or analyzed during the current study are available from the corresponding author on reasonable request.

\section{Authors' contributions}

$\mathrm{XL}, \mathrm{JZ}$ and YF finished most of the experiments and wrote the manuscript. JW and HZ performed the FACS. FD constructed the stable cell lines. BJ, JW and BN designed the experiments and revised the manuscript. All authors read and approved the final manuscript.

\section{Ethics approval and consent to participate}

Not applicable.

\section{Patient consent for publication}

Not applicable

\section{Competing interests}

The authors declare that they have no competing interests.

\section{References}

1. Jemal A, Bray F, Center MM, Ferlay J, Ward E and Forman D: Global cancer statistics. CA Cancer J Clin 61: 69-90, 2011.

2. Asghar U and Meyer T: Are there opportunities for chemotherapy in the treatment of hepatocellular cancer? J Hepatol 56: 686-695, 2012

3. Sukowati CH, Rosso N, Crocè LS and Tiribelli C: Hepatic cancer stem cells and drug resistance: Relevance in targeted therapies for hepatocellular carcinoma. World J Hepatol 2: 114-126, 2010.

4. Wong $\mathrm{H}$ and Schotz MC: The lipase gene family. J Lipid Res 43 993-999, 2002

5. Santamarina-Fojo S, González-Navarro H, Freeman L, Wagner E and Nong Z: Hepatic lipase, lipoprotein metabolism, and atherogenesis. Arterioscler Thromb Vasc Biol 24: 1750-1754, 2004.

6. Nomura DK, Long JZ, Niessen S, Hoover HS, Ng SW and Cravatt BF: Monoacylglycerol lipase regulates a fatty acid network that promotes cancer pathogenesis. Cell 140: 49-61, 2010

7. Ding Q, Chang CJ, Xie X, Xia W, Yang JY, Wang SC, Wang Y, Xia J, Chen L, Cai C, et al: APOBEC3G promotes liver metastasis in an orthotopic mouse model of colorectal cancer and predicts human hepatic metastasis. J Clin Invest 121: 4526-4536, 2011.

8. Galluzzi L, Goubar A, Olaussen KA, Vitale I, Senovilla L, Michels J, Robin A, Dorvault N, Besse B, Validire P, et al Prognostic value of LIPC in non-small cell lung carcinoma. Cell Cycle 12: 647-654, 2013.
9. Ma S, Lee TK, Zheng BJ, Chan KW and Guan XY: CD133+ HCC cancer stem cells confer chemoresistance by preferential expression of the Akt/PKB survival pathway. Oncogene 27: 1749-1758, 2008.

10. Galluzzi L, Senovilla L, Vitale I, Michels J, Martins I, Kepp O, Castedo M and Kroemer G: Molecular mechanisms of cisplatin resistance. Oncogene 31: 1869-1883, 2012.

11. Livak and Schmittgen: Analysis of relative gene expression data using real-time quantitative PCR and the 2(-Delta Delta C(T)) Method. Methods 25: 402-408, 2001.

12. Franken NA, Rodermond HM, Stap J, Haveman J and van Bree C: Clonogenic assay of cells in vitro. Nat Protoc 1: 2315-2319, 2006.

13. Stein W, Bohner J and Bahlinger M: Macro lipase - a new member of the family of immunoglobulin-linked enzymes. J Clin Chem Clin Biochem 25: 837-843, 1987.

14. Muñoz-Perez M, Sarrion-Pelous D, Jimenez-Jimenez J, MartinezMontiel P and Gallego-Valdes M: Chronic increased serum lipase in a patient with suspected pancreatic adenocarcinoma. Clin Chem 43: 191-193, 1997.

15. Diani G, Poma G, Novazzi F, Zanirato S, Porta C, Moroni M, Melzi d' Eril GV and Moratti R: Increased serum lipase with associated normoamylasemia in cancer patients. Clin Chem 44: 1043-1045, 1998.

16. Singh SK, Clarke ID, Terasaki M, Bonn VE, Hawkins C, Squire J and Dirks PB: Identification of a cancer stem cell in human brain tumors. Cancer Res 63: 5821-5828, 2003.

17. Fang D, Nguyen TK, Leishear K, Finko R, Kulp AN, Hotz S, Van Belle PA, Xu X, Elder DE and Herlyn M: A tumorigenic subpopulation with stem cell properties in melanomas. Cancer Res 65: 9328-9337, 2005 .

18. Collins AT, Berry PA, Hyde C, Stower MJ and Maitland NJ: Prospective identification of tumorigenic prostate cancer stem cells. Cancer Res 65: 10946-10951, 2005.

19. Miki J, Furusato B, Li H, Gu Y, Takahashi H, Egawa S, Sesterhenn IA, McLeod DG, Srivastava S and Rhim JS: Identification of putative stem cell markers, CD133 and CXCR4 in hTERT-immortalized primary nonmalignant and malignant tumor-derived human prostate epithelial cell lines and in prostate cancer specimens. Cancer Res 67: 3153-3161, 2007.

20. O'Brien CA, Pollett A, Gallinger S and Dick JE: A human colon cancer cell capable of initiating tumour growth in immunodeficient mice. Nature 445: 106-110, 2007.

21. Ricci-Vitiani L, Lombardi DG, Pilozzi E, Biffoni M, Todaro M, Peschle $C$ and De Maria R: Identification and expansion of human colon-cancer-initiating cells. Nature 445: 111-115, 2007.

22. Suetsugu A, Nagaki M, Aoki H, Motohashi T, Kunisada T and Moriwaki H: Characterization of CD133+ hepatocellular carcinoma cells as cancer stem/progenitor cells. Biochem Biophys Res Commun 351: 820-824, 2006.

23. Yin S, Li J, Hu C, Chen X, Yao M, Yan M, Jiang G, Ge C, Xie H, Wan D, et al: CD133 positive hepatocellular carcinoma cells possess high capacity for tumorigenicity. Int J Cancer 120: 1444-1450, 2007.

24. Bao S, Wu Q, Sathornsumetee S, Hao Y, Li Z, Hjelmeland AB, Shi Q, McLendon RE, Bigner DD and Rich JN: Stem cell-like glioma cells promote tumor angiogenesis through vascular endothelial growth factor. Cancer Res 66: 7843-7848, 2006.

25. Shmelkov SV, Butler JM, Hooper AT, Hormigo A, Kushner J, Milde T, St Clair R, Baljevic M, White I, Jin DK, et al: CD133 expression is not restricted to stem cells, and both $\mathrm{CD} 133^{+}$and CD133- metastatic colon cancer cells initiate tumors. J Clin Invest 118: 2111-2120, 2008.

26. Meng X, Li M, Wang X, Wang Y and Ma D: Both $\mathrm{CD}_{133^{+}}$and CD133- subpopulations of A549 and H446 cells contain cancerinitiating cells. Cancer Sci 100: 1040-1046, 2009.

27. Jaksch M, Múnera J, Bajpai R, Terskikh A and Oshima RG: Cell cycle-dependent variation of a CD133 epitope in human embryonic stem cell, colon cancer, and melanoma cell lines. Cancer Res 68: 7882-7886, 2008.

28. Yang JD, Nakamura I and Roberts LR: The tumor microenvironment in hepatocellular carcinoma: Current status and therapeutic targets. Semin Cancer Biol 21: 35-43, 2011.

29. Cheung ST, Leung KL, Ip YC, Chen X, Fong DY, Ng IO, Fan ST and So S: Claudin-10 expression level is associated with recurrence of primary hepatocellular carcinoma. Clin Cancer Res 11: 551-556, 2005.

30. Ip YC, Cheung ST, Lee YT, Ho JC and Fan ST: Inhibition of hepatocellular carcinoma invasion by suppression of claudin-10 in HLE cells. Mol Cancer Ther 6: 2858-2867, 2007.

31. Suh Y, Yoon CH, Kim RK, Lim EJ, Oh YS, Hwang SG, An S, Yoon G, Gye MC, Yi JM, et al: Claudin-1 induces epithelial-mesenchymal transition through activation of the c-Abl-ERK signaling pathway in human liver cells. Oncogene 32: 4873-4882, 2013. 